\title{
Safety of Novel Targeted Therapies in Oncology
}

\author{
Rashmi R. Shah ${ }^{1}$ - Giuseppe Curigliano ${ }^{2,3}$
}

Published online: 16 January 2019

(c) Springer Nature Switzerland AG 2019

Advances in cell biology and genomics have uncovered a number of oncogenic pathways and identified unique molecular signatures of a variety of cancers previously treatable only with relatively non-specific chemotherapeutic agents. Coupled with corresponding advances in medicinal chemistry, oncologists now have at their disposal highly targeted/ specific therapies that can be personalised to suit individual patients [1-6].

While these novel agents have improved the outcomes of cancer therapy, especially in terms of progression-free survival, they are also associated with disabling, serious or potentially fatal, adverse effects which may impact heavily on patient's quality of life. Some of these adverse effects are on-target effects closely associated with therapeutic response, making their management challenging if the patient is otherwise responding to treatment, while others are off-target effects. Clinical experience has shown that in the event of an intolerable adverse effect, it may be possible to switch the patient from one member to another in the same pharmacotherapeutic class. This calls for a better understanding of the pharmacokinetics and mechanisms that underpin efficacy and toxicity, as well as the relative safety profiles of the drugs within a class. The presence or emergence of resistance to a first-line (and later second-line) agent is another important consideration that determines the choice of a drug. There is therefore a need for a single resource that physicians can refer to with regard to these issues for each pharmacotherapeutic class.

Part of a theme issue on "Safety of Novel Anticancer Therapies: Future Perspectives". Guest Editors: Rashmi R. Shah, Giuseppe Curigliano.

Rashmi R. Shah

clinical.safety@hotmail.co.uk

8 Birchdale, Gerrards Cross, Buckinghamshire, UK

2 Division of Early Drug Development for Innovative Therapy, IEO, European Institute of Oncology IRCCS, Milan, Italy

3 Department of Oncology and Haematology (DIPO), University of Milan, Milan, Italy
In order to ensure that the resource is as current as practical, a themed issue of a journal, rather than a book, is clearly the most appropriate medium. This issue of Drug Safety includes reviews of the safety and tolerability of novel small molecule and targeted therapies in oncology across a range of pharmacotherapeutic classes.

Procaccio and colleagues [7] reviewed approved as well as investigational antiangiogenic and vascular disrupting agents in the treatment of gastrointestinal cancers, focusing on their pharmacology, safety profiles, promising predictive biomarkers and future perspectives of combination therapies that include these agents. The safety of approved epidermal growth factor receptor (EGFR) inhibitors, as well as those in the pipeline, has been reviewed in detail by Shah and Shah [8], who also focus on agents discontinued from development and the challenges arising from the emergence of resistance to these agents. Rocco et al. [9] provide a comprehensive state-of-the-art review of the pharmacology, safety and tolerability profiles of currently available ALK inhibitors in non-small cell lung cancer. Following a discussion on the emergence of resistance to these agents, they too go on to review several third-generation $A L K$ inhibitors that are currently under investigation. The safety of the two currently approved c-MET inhibitors (cabozantinib and crizotinib) has been reviewed by Puccini and colleagues [10], who also go on to consider novel promising c-MET inhibitors and novel targeted combination strategies under development in different cancer types. They focus on their safety profile, making a plea for improvements in biomarker-driven patient selection to optimise outcomes. In another article, Shah [11] highlights the safety profile of HDAC inhibitors, especially their cardiac adverse effects.

Curigliano and Shah [12] review the safety of approved PI3K inhibitors, as well as those under development and those that have been discontinued, highlighting their immune-mediated cutaneous reactions, severe diarrhoea with or without colitis, hepatotoxicity and pneumonitis, and their risk/benefit when used in combination with other agents. Carpenter and Ray [13] review the hedgehog pathway and the toxicity associated with its inhibitors, pointing 
out the development of resistance to vismodegib and sonidegib, leading to a long list of agents under development to target the sonic hedgehog pathway. Baraibar and colleagues [14] address the safety of immune checkpoints inhibitors (PD-1 and PD-L1) in cancer and report that enhancement of the immune system response by immunotherapy has stimulated a paradigm shift in the treatment of malignancies, but noting that this shift in the balance of the immune system can also produce side effects that involve multiple organs. They emphasise the different toxicity patterns of these agents, with little experience in recognising and managing their toxicity. Antibody-drug conjugates are expected to deliver and release cytotoxic agents at the tumour site with reduced systemic toxicity. Wolska-Washer and Robak [15] outline the current status of these conjugates in the treatment of malignant diseases and emphasise the challenges in designing a clinically adequate conjugate, while Wolf and colleagues [16] review the safety of adoptive cell therapy and conclude that treatment-related toxicities represent a major hurdle in the wider implementation of this therapeutic modality, and call for an international consensus for the management of toxicity of adoptive cell therapy.

Readers should find the contents of this issue interesting and informative, as well as of practical clinical value.

\section{Compliance with Ethical Standards}

This is a review of data in the public domain and the authors declare compliance with all ethical standards.

Funding No sources of funding were used to assist in the preparation of this editorial.

Conflict of interest Rashmi Shah was formerly a Senior Clinical Assessor at the Medicines and Healthcare products Regulatory Agency (MHRA), London, UK, and now provides expert consultancy services to a number of pharmaceutical companies. Giuseppe Curigliano has received consulting fees from Pfizer, Novartis, Eli Lilly and Roche; travel support from Roche; and lecture payments from Pfizer. Rashmi Shah and Giuseppe Curigliano have no other conflicts of interest that are relevant to the content of this editorial.

\section{References}

1. Weng L, Zhang L, Peng Y, Huang RS. Pharmacogenetics and pharmacogenomics: a bridge to individualized cancer therapy. Pharmacogenomics. 2013;14:315-24.
2. Tsimberidou AM, Iskander NG, Hong DS, Wheler JJ, Falchook GS, Fu S, et al. Personalized medicine in a phase I clinical trials program: the MD Anderson Cancer Center initiative. Clin Cancer Res. 2012;18:6373-83.

3. Tsimberidou AM. Targeted therapy in cancer. Cancer Chemother Pharmacol. 2015;76:1113-32.

4. Wheler JJ, Janku F, Naing A, Li Y, Stephen B, Zinner R, et al. Cancer therapy directed by comprehensive genomic profiling: a single center study. Cancer Res. 2016;76:3690-701.

5. Sánchez NS, Mills GB, Mills Shaw KR. Precision oncology: neither a silver bullet nor a dream. Pharmacogenomics. 2017;18:1525-39.

6. Heitzer E, Haque IS, Roberts CES, Speicher MR. Current and future perspectives of liquid biopsies in genomics-driven oncology. Nat Rev Genet. (Epub 8 Nov 2018).

7. Procaccio L, Damuzzo V, Di Sarra F, Russi A, Todino F, Dadduzio V, et al. Safety and tolerability of anti-angiogenic protein kinase inhibitors and vascular disrupting agents in cancer: focus on gastrointestinal malignancies. Drug Saf. 2019. https://doi. org/10.1007/s40264-018-0776-6.

8. Shah RR, Shah DR. Safety and tolerability of epidermal growth factor receptor (EGFR) tyrosine kinase inhibitors in oncology. Drug Saf. 2019. https://doi.org/10.1007/s40264-018-0772-x.

9. Rocco D, Battiloro C, Della Gravara L, Gridelli C. Safety and tolerability of ALK inhibitors in non-small cell lung cancer. Drug Saf. 2019. https://doi.org/10.1007/s40264-018-0771-y.

10. Puccini A, Marín-Ramos NI, Bergamo F, Schirripa M, Lonardi S, Lenz H-J, et al. Safety and tolerability of c-MET inhibitors in cancer. Drug Saf. 2019. https://doi.org/10.1007/s40264-018-0780-x.

11. Shah RR. Safety and tolerability of histone deacetylase (HDAC) inhibitors in oncology. Drug Saf. 2019. https://doi.org/10.1007/ s40264-018-0773-9.

12. Curigliano G, Shah RR. Safety and tolerability of phosphatidylinositol 3-kinase (PI3K) inhibitors in oncology. Drug Saf. 2019. https://doi.org/10.1007/s40264-018-0778-4.

13. Carpenter RL, Ray H. Safety and tolerability of sonic hedgehog pathway inhibitors in cancer. Drug Saf. 2019. https://doi. org/10.1007/s40264-018-0777-5.

14. Baraibar I, Melero I, Ponz-Sarvise M, Castanon E. Safety and tolerability of immune checkpoints inhibitors (PD-1 and PD-L1) in cancer. Drug Saf. 2019. https://doi.org/10.1007/s4026 4-018-0774-8.

15. Wolska-Washer A, Robak T. Safety and tolerability of antibody-drug conjugates in cancer. Drug Saf. 2019. https://doi. org/10.1007/s40264-018-0775-7.

16. Wolf B, Zimmermann S, Arber C, Irving M, Trueb L, Coukos G. Safety and tolerability of adoptive cell therapy in cancer. Drug Saf. 2019. https://doi.org/10.1007/s40264-018-0779-3. 